\title{
Properties and parameters for effective laser hair removal: A review
}

\section{Sara Gonçalves}

\author{
Clínica Áurea - Aesthetic Biomedicine Clinic; Rua de Três Lagares, bloco 1, loja G, 5000-577 Vila Real, Portugal \\ Corresponding author: Sara Gonçalves, MSc, E-mail: sgoncalves@utad.pt
}

\begin{abstract}
Because some people seek consultations for the removal of unwanted body hair, a permanent, safe, and non-invasive method of hair removal is needed. Laser hair removal has been a popular cosmetic procedure. It is a monthly treatment that may take up to ten sessions for permanent results. However, its efficacy varies due to the patient's biological skin hair traits, the adjustment of the optimal parameters of the laser, and the protocols. The purpose of this review is to discuss the properties and parameters employed for laser hair removal. Once parameters for laser hair removal have been optimized, this treatment offers an effective and permanent solution for unwanted body hair.
\end{abstract}

Key words: Laser hair removal; Fluence; Frequency; Pulse width; Skin type

\section{INTRODUCTION}

Unwanted or excess hair is a prevalent condition affecting various aspects of life, including medical, social, and cultural. Because people tend to seek consultations for the removal of unwanted body hair, a permanent, safe, and non-invasive method is needed. Laser hair removal has been a popular cosmetic procedure. It is a monthly treatment that may take up to twelve sessions for permanent results [1]. Results may be understood as the elimination of hair or a decrease in the density of hair follicles or their diameter. Laser hair removal may be employed in preparation for surgery [2], for hirsutism [3], and to treat scars [4], folliculitis, and hypertrichosis. A correct initial diagnosis is necessary for optimal results [5]. These depend upon factors that affect the hair cycle, such as the location of hair growth, the sex, the age, and the season of the year; as well as factors that influence hair growth, such as genetic predisposition, fetal development, medications taken, and other hormonal, nutritional, and psychological factors.

However, the efficacy varies due to the patient's biological skin hair traits, the adjustment of the optimal parameters of the laser, and the protocols.
The purpose of this review is to discuss the properties and parameters employed for laser hair removal.

\section{LASER PROPERTIES AND PARAMETERS}

Simply put, a laser is a light-producing device with an active medium-which may be liquid, solid, or gaseous-within a cavity, called the optical cavity, bounded by two mirrors (Fig. 1). When the proportion of the excited atoms is increased, exceeding the medium and raised to a higher energy level, the LASER (light amplification stimulated by emissions of radiation) effect is produced inside the cavity. The light escapes from the cavity through a partial reflection mirror, continuously or in pulses, and has specific properties:

- Coherence (all photons follow the same direction);

- Monochromacy (all photons are emitted with the same wavelength, which makes the laser beam onecolor);

- Collimation (the waves have the same direction and the light is parallel, not divergent, narrow, and concentrated);

- Small area of focus: the light may be focused on a very small area, allowing it to reach an extremely high luminous intensity (Fig. 2).

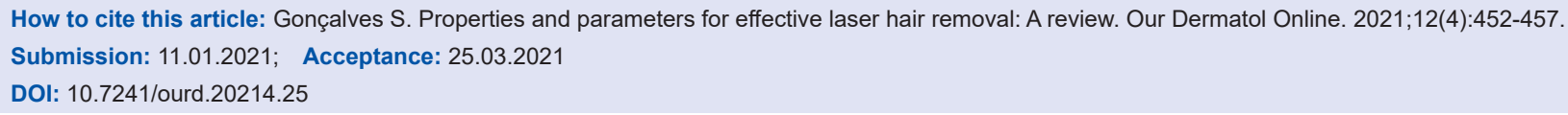




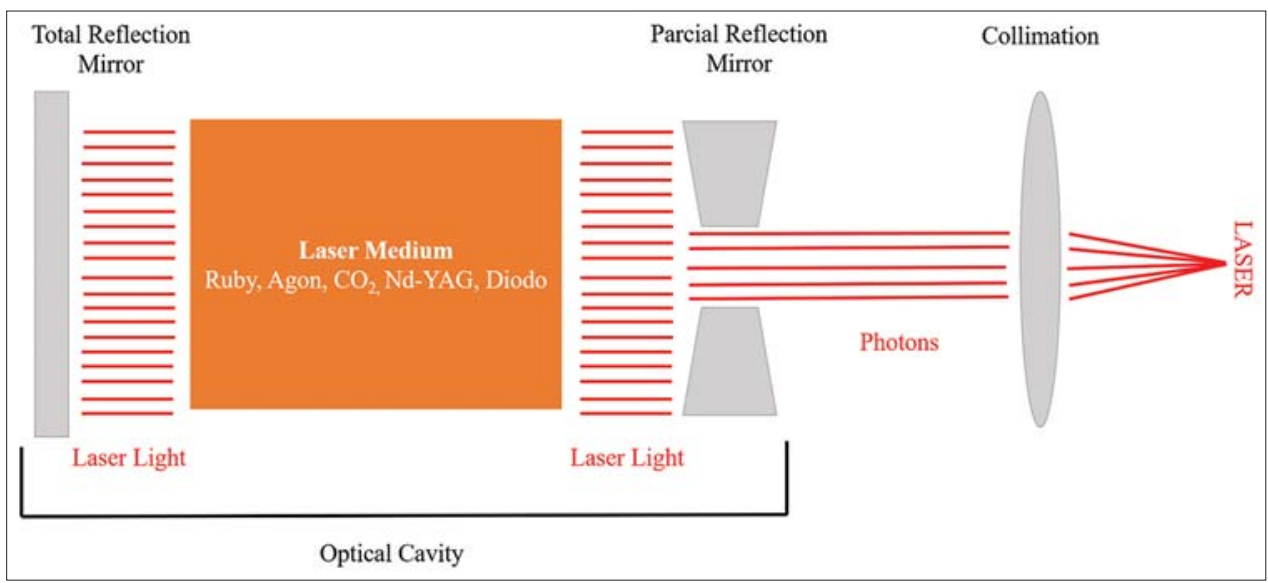

Figure 1: Laser scheme.

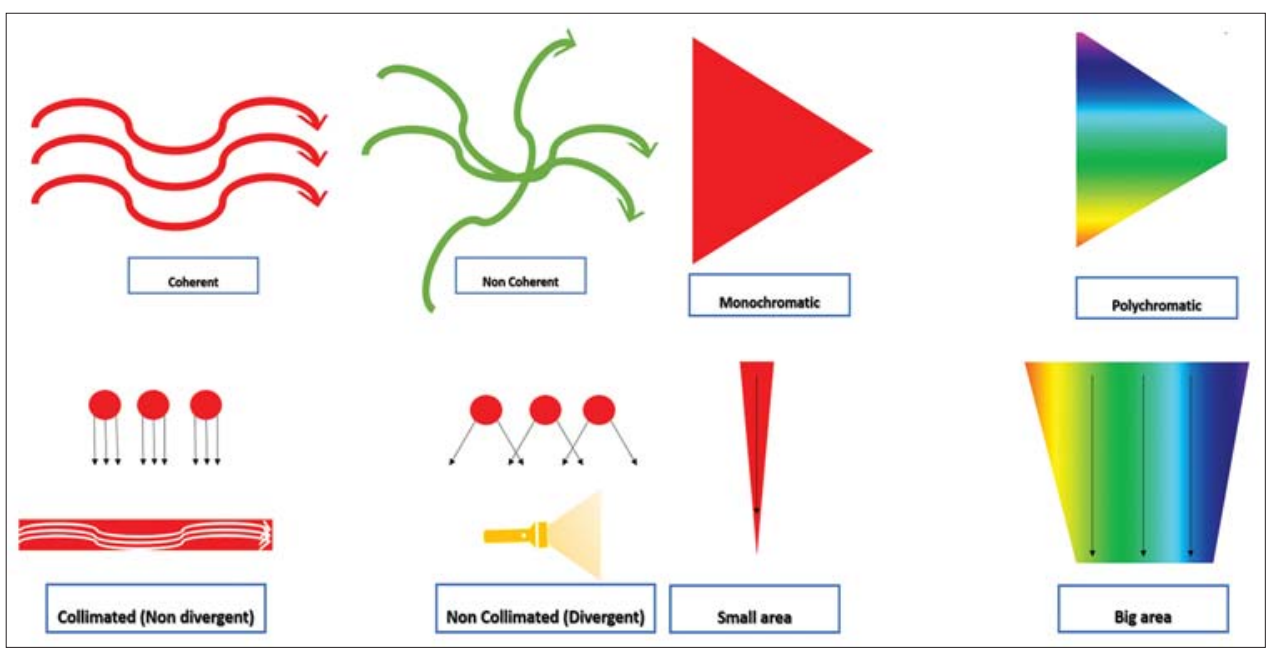

Figure 2: Properties of LASER light.

When laser light falls on the skin it may be reflected, transmitted, dispersed, or absorbed. However, it only produces an effect on tissues if absorbed. Absorption happens because of the presence of chromophores in the skin-elements that selectively absorb specific wavelengths. Energy transmitted into the dermis eliminates chromophores; that is, it fragments the molecules of a chromophore into smaller particles without damage to the surrounding tissue and with minimal epidermal damage.

Laser hair removal is a process that involves complex selective photothermolysis via the epidermis-dermis matrix, aimed to cause hair follicle damage while sparing the epidermis. The thermal action that causes the hair removal effect is caused by heat accumulation in the dermis [1] under strict safety and preservation parameters for the epidermis (Fig. 3). Prolonged exposure to a temperature above $45^{\circ} \mathrm{C}$ causes the destruction of the hair follicle [2], with the consequent elimination of the hair in that area [3].
Lasers carry various characteristics:

- Wavelength: a band from the electromagnetic spectrum from which a laser emits its light; wavelength is chosen according to the depth to be reached and the chromophore in the target tissue;

- Emission type: the method of energy supplying: continuous or pulsed; continuous emission is a constant energy supply, while pulsed is the application of the energy in intervals and is further divided into:

o Semicontinuous: pulsed emission at a frequency equivalent to continuous emission;

o Multipulsed: emission of a "train" of pulses (two, three, or more);

o Superpulsed: emission that, by means of electronic devices, is able to generate highpower pulses with emission times as small as microseconds;

o Q-switched: exceedingly high-power pulses and short emission times, as small as nanoseconds or femtoseconds [6]; 


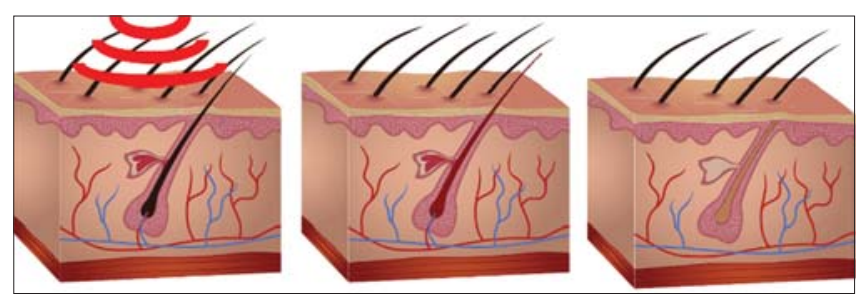

Figure 3: Principle of action of selective photothermolysis.

- Pulse duration: the time that the light takes to emit, measured in picoseconds, nanoseconds, microseconds, or milliseconds:

o Thermal Relaxation Time (TRT): the time that the tissue needs to reduce the temperature generated by the emission of the laser light by 50\%; the pulse duration must not be below the TRT; this way, less energy dissipation in the underlying structures and no undesirable effects, such as epidermic damage, happen [7]:

$\square$ TRT for the epidermis: 3-10 ms;

$\square$ TRT for the hair follicle: $40-100 \mathrm{~ms}$;

o Thermal Damage Time (TDT): the time required to heat the target chromophore;

- Pulse frequency: the number of pulses of laser light emitted per second, measured in hertz $(\mathrm{Hz})$;

- Delay: the time between each pulse; especially important parameter related to the TRT;

- Fluence: the relation between the energy supplied by the light emitter and the surface of the radiation beam-not of the total area of treatmentmeasured in joules per square centimeter $\left(\mathrm{J} / \mathrm{cm}^{2}\right)$; this is the energy that heats;

- Irradiance: the relationship between the actual output power of the light emitter and the surface of the radiation beam-not of the total area of treatment-measured in watts per square centimeter $\left(\mathrm{W} / \mathrm{cm}^{2}\right)$;

- Spot: the size of the irradiation beam at the point of application; although not an adjustable parameter of a laser machine, it is equally as important; the spot is the size of the crystal through which the laser light escapes and large spots sizes are associated with greater power; one must pay attention to note that a spot of $2100 \mathrm{~W}$ and $1 \mathrm{~cm}^{2}$ is different from a spot of $2100 \mathrm{~W}$ and $6 \mathrm{~cm}^{2}$, with small spots more suitable for the face and large spots for the body, as smaller spots lead to greater dispersion and a lower energy density (Fig. 4a), and larger spots lead to less dispersion and a higher energy density (Fig. 4b).

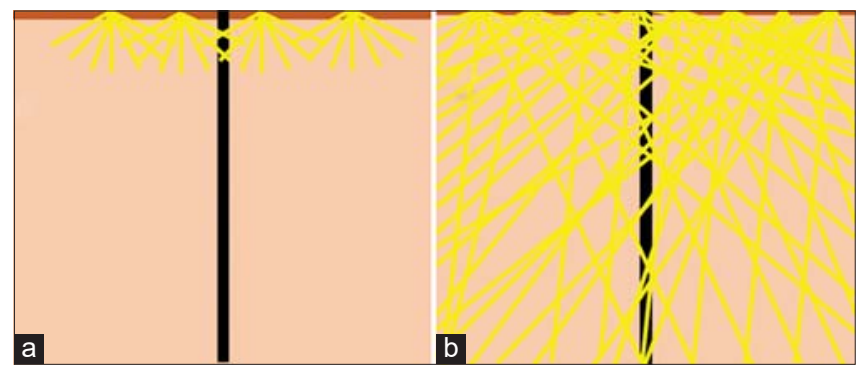

Figure 4: $(a$ and $b)$ Difference between small and large spot sizes.

\section{Effective Hair Removal}

Two types of factors are involved in effective hair removal: patient-dependent and laser-dependent [8].

The patient-dependent factors include:

- Amount of melanin in the hair follicle: As melanin is the target of the laser light, the higher its concentration and the closer it is to the capillary matrix, the more effective the treatment will be. For this reason, gray or less pigmented hairs are unaffected by the laser [9].

- Amount of melanin in the skin: Melanin is also found in the epidermis. Epidermal melanin competitively absorbs the same wavelengths as employed for hair removal. In darker-skinned individuals, the greater epidermal melanin content competes with the hair follicle for light absorption, increasing the risk of thermal blisters and hyperpigmentation. It is important to determine the skin's phototype and choose the appropriate laser parameters. The darkest phototypes are those with the highest risk of burn and they require a laser with a longer wavelength and a longer pulse duration.

- Hair thickness: The thicker and more pigmented the hair, the greater the energy absorption and the better results from laser hair removal. Fine hairs absorb less energy and results take longer to appear.

- Depth of the hair follicle: It is important to consider this aspect with lasers reaching certain depths. For more superficial hair, such as facial, lasers of shorter wavelengths are employed; for deeper hair, such as that of the back in most males, longer wavelengths are employed.

- Growth stage: During the anagen phase, the hair is thicker and more pigmented, making it the most appropriate phase to perform laser hair removal.

The laser-dependent factors include:

- Wavelength: The selection of the most appropriate laser wavelength is essential. 
o Lighter skin types respond well to shorter wavelengths, while darker skin types respond well to longer wavelengths.

o The hair follicle may reside between 2 to $7 \mathrm{~mm}$ below the surface of the skin, so different wavelengths are required for the best results.

o Light-colored and fine hair requires a laser with a high attraction to melanin, while darker and thicker hair will respond well to a laser with a low attraction to melanin [10].

- Pulse duration: An appropriate pulse duration is essential and allows the professional to reach hairs of different diameters. Longer pulses are often needed as it is necessary to heat the hair follicle to the outermost portion and not only to carbonize the hair rod. In the beginning, the ideal pulse duration was thought to be between the TRT of the epidermis and that of the hair follicle. However, fine hair heats up more quickly than thicker hair, yet it is unable to hold the heat and, thus, requires short pulse durations (5-10 ms), while thicker hair requires longer pulse durations (40-60 ms).

- Spot size: A larger spot means higher penetration and higher efficiency. A small spot size causes the photons to scatter radially not reaching the hair bulbs, leading to greater dispersion and a decreased fluence. The spot size should be larger than the depth of the light penetrating the tissue, namely 5-10 nm [11].

- Pulse frequency: The number of shots per second that helps to reduce the time of the treatment session.
- Delay: A larger delay leads to the epidermis protecting itself more effectively.

- Number of pulses: Splitting the energy into multiple pulses enables the application of a higher fluence and more effectively protects the epidermis.

- Fluence: The efficiency is proportional to the fluence. If the fluence is too high, heat is produced in excess, leading to skin burns. A too low of a fluence may lead to a temporary hair loss lasting one to three months. Permanent hair reduction requires high-quality lasers that are capable of generating large amounts of energy, ensuring irreversible damage to the hair.

\section{Pre- and Post-Treatment Recommendations}

Complying with the following recommendations is of utmost importance to obtain satisfactory results from laser hair removal.

The pre-treatment recommendations are the following [8]:

1. Remove make-up from the areas to be treated: Makeup prevents the laser from reaching the hair root, compromising the results.

2. Do not apply creams or deodorants to the areas to be treated: Creams and deodorants prevent the correct application of the ultrasound gel, in addition to the fact that, after the action of the laser, it sticks, hindering the progression of the handle.

3. Cut the hair in the areas to be treated: It is advisable for patients to cut their hair at home with a razor and depilatory cream if needed.

Table 1: Laser parameters from studies: fluence, pulse width, and frequency

\begin{tabular}{|c|c|c|c|c|c|}
\hline Year & Skin Type & Fluence $\left(\mathrm{J} / \mathrm{cm}^{2}\right)$ & Pulse Width (ms) & Frequency (Hz) & Reference \\
\hline \multirow[t]{2}{*}{2011} & IV-V & $5-8$ & 20 & 10 & {$[12]$} \\
\hline & & $25-35$ & 30 & 2 & \\
\hline 2012 & II-V & 15 & 15 & 5 & [13] \\
\hline 2012 & I-IV & $25-35$ & 30 & $N / A$ & [14] \\
\hline 2012 & N/A & $7-9$ & 10 & 10 & [15] \\
\hline 2012 & III-IV & $5-10$ & 20 & 10 & [16] \\
\hline 2013 & IV & 40 & 20 & 1 & [17] \\
\hline \multirow[t]{2}{*}{2013} & IV-VI & 24 & 6 & 10 & [18] \\
\hline & & 10 & 20 & & \\
\hline 2013 & N/A & 30 & 12 & $N / A$ & [19] \\
\hline \multirow[t]{2}{*}{2014} & II-V & $6-12$ & 20 & $\mathrm{~N} / \mathrm{A}$ & [20] \\
\hline & & $6-12$ & $30-70$ & & \\
\hline 2015 & II-IV & $25-33$ & $30-45$ & $N / A$ & [21] \\
\hline 2016 & $\mathrm{~N} / \mathrm{A}$ & 60 & $30-80$ & $\mathrm{~N} / \mathrm{A}$ & [22] \\
\hline 2016 & $I V-V I$ & $3-10$ & $N / A$ & 10 & [23] \\
\hline 2016 & III-V & 10 & 30 & 10 & [24] \\
\hline \multirow[t]{2}{*}{2018} & IV & $26-30$ & 30 & $\mathrm{~N} / \mathrm{A}$ & [25] \\
\hline & V & $22-26$ & & & \\
\hline \multirow[t]{3}{*}{2020} & III & 24 & 30 & 1 & [26] \\
\hline & IV & 22 & & & \\
\hline & V & 20 & & & \\
\hline
\end{tabular}


4. Between the sessions, only cut the hair: Plucking the hair at the root makes laser hair removal less effective. Patients who have had their hair removed must wait 15 to 21 days before the next session.

5. Always use a body scrub before each session: This helps to unravel hair.

The post-treatment recommendations are the following:

1. Avoid sun exposure in the following 48 hours and indoor tanning 5 days after treatment: The skin after laser hair removal is especially sensitive and the pores are open. Sunlight and indoor tanning may lead to hyperpigmentation.

2. Use high-SPF sunscreen and protect the treated area from the sun. Tanning after a session may increase melanin regeneration, leading to in hyperpigmentation.

3. Minimize the use of makeup immediately after the treatment: The face is especially sensitive after having come in contact with laser light.

4. Moisturize the treated areas: With each session, the skin will acquire an increasingly better appearance. The use of moisturizer accelerates this process.

\section{Parameters from Studies}

The PubMed database was used. An initial query yielded 52 results and, after filtering these against the last ten years, 24 results. After reading the abstracts, 15 studies remained, eliminating 9. Table 1 shows all the parameters gathered.

\section{CONCLUSION}

Although the ideal patient is one with light skin and dark hair, this is not always the case. Parameters need to be adjusted to the patient's characteristics. The operator of a laser hair removal machine needs to perform a correct evaluation of the patient, determining the presence of a health condition that might compromise the results, but also assessing the skin phototype and the hair color correctly to optimize the parameters (Table 1).

The fluence, pulse frequency, and pulse duration seem to play an important role. The authors recommend a high fluence $\left(20-30 \mathrm{~J} / \mathrm{cm}^{2}\right)$, a high frequency $(10 \mathrm{~Hz})$, and a medium pulse width $(30 \mathrm{~ms})$. Once the frequency is set on the machine, fluence and pulse width will automatically adjust since they are linked to one another. A high frequency requires a low pulse width and fluence, and a low frequency allows for a higher pulse width and fluence. Most machines are equipped with predefined values, parameters ideal for an operator new to the machine. However, it is important to adjust the parameters from session to session for the most satisfactory outcome.

Because parameters for laser hair removal continue to evolve as further understanding is being reached, further investigation on the optimization of these parameters is needed.

\section{Ethics Statement}

The procedures followed were in accordance with the ethical standards of the responsible Committee on Human Experimentation (institutional and national) and the Helsinki Declaration of 1975, as revised in 2000 and 2008.

\section{REFERENCES}

1. Casey AS, Goldberg D. Guidelines for laser hair removal. J Cosmet Laser Ther. 2008;10:24-33.

2. Srihari RS, Naveen AM, Sreekar $H$. The limberg flap reconstruction - The optimal surgery for pilonidal sinus disease. Our Dermatol Online. 2016;7:271-5.

3. Martini L. The tale of the apewoman who craved to become a siren: A natural way to combat radically hirsutism. Our Dermatol Online. 2017;8:152-5.

4. Bimbi C, Brzeziński P. Combined treatment of keloids and scars with Nd:YAG $1064 \mathrm{~nm}$ laser and cryotherapy: Report of clinical cases. Our Dermatol Online 2020;11:149-53.

5. Gonçalves S. A Consulta de Biomedicina Estética. vol. 1. 1st ed. Portugal: escrytos; 2021.

6. Vidurrizaga C. Medicina Estética - Abordaje terapêutico. n.d.

7. Lepselter J, Elman M. Biological and clinical aspects in laser hair removal. J Dermatol Treat. 2004;15:72-83.

8. Gonçalves S. A Depilação a Laser. vol. 1. 1st ed. Portugal: escrytos; n.d.

9. Gan S, Graber E. Laser hair removal: A review. Dermatol Surg. 2013;39:823-38.

10. Hair Removal - Lasers Explained. Dr Nathan Holt n.d. https:// cambridgelaserclinic.com/laser-treatments/hair-removal/lasersexplained/ (accessed January 18, 2021).

11. Sadighha A, Mohaghegh Zahed G. Meta-analysis of hair removal laser trials. Lasers Med Sci. 2009;24:21-5.

12. Pai GS, Bhat PS, Mallya H, Gold M. Safety and efficacy of lowfluence, high-repetition rate versus high-fluence, low-repetition rate 810 -nm diode laser for permanent hair removal - A split-face comparison study. J Cosmet Laser Ther. 2011;13:134-7.

13. Barolet D. Low fluence-high repetition rate diode laser hair removal 12-month evaluation: Reducing pain and risks while keeping clinical efficacy. Lasers Surg Med. 2012;44:277-81.

14. Halachmi S, Lapidoth M. Low-fluence vs. standard fluence hair removal: A contralateral control non-inferiority study. J Cosmet Laser Ther. 2012;14:2-6.

15. Wanitphakdeedecha R, Thanomkitti K, Sethabutra P, Eimpunth S, Manuskiatti W. A split axilla comparison study of axillary hair removal with low fluence high repetition rate $810 \mathrm{~nm}$ diode laser 


\section{www.odermatol.com}

vs. high fluence low repetition rate $1064 \mathrm{~nm}$ Nd:YAG laser. J Eur Acad Dermatol Venereol. 2012;26:1133-6.

16. Chen J, Liu XJ, Huo MH. Split-leg comparison of low fluence diode laser and high fluence intense pulsed light in permanent hair reduction in skin types III to IV. Australas J Dermatol. 2012;53:186-9.

17. Fontana CR, Bonini D, Bagnato VS. A 12-month follow-up of hypopigmentation after laser hair removal. J Cosmet Laser Ther. 2013;15:80-4.

18. Lapidoth M, Adatto M, Cohen S, Ben-Amitai D, Halachmi S. Hypertrichosis in Becker's nevus: Effective low-fluence laser hair removal. Lasers Med Sci. 2014;29:191-3.

19. Bodendorf MO, Wagner JA, Grunewald S, Simon J-C, Paasch U. Efficacy and safety of laser shields to prevent radiant transmission onto pigmented nevi during laser epilation: An ex vivo histology study. Int J Hyperthermia. 2013;29:539-43.

20. Koo B, Ball K, Tremaine A-M, Zachary CB. A comparison of two 810 diode lasers for hair removal: Low fluence, multiple pass versus a high fluence, single pass technique. Lasers Surg Med. 2014;46:270-4.

21. Jo SJ, Kim JY, Ban J, Lee Y, Kwon O, Koh W. Efficacy and safety of hair removal with a long-pulsed diode laser depending on the spot size: A randomized, evaluators-blinded, left-right study. Ann Dermatol. 2015;27:517-22.

22. Courtney E, Goldberg D. Clinical evaluation of hair removal using an $810 \mathrm{~nm}$ diode laser with a novel scanning device. J Drugs
Dermatol. 2016;15:1330-3.

23. Agarwal M, Velaskar S, Gold MH. Efficacy of a low fluence, high repetition rate $810 \mathrm{~nm}$ diode laser for permanent hair reduction in Indian patients with skin types IV-VI. J Clin Aesthetic Dermatol. 2016;9:29-33.

24. Li W, Liu C, Chen Z, Cai L, Zhou C, Xu Q, et al. Safety and efficacy of low fluence, high repetition rate versus high fluence, low repetition rate 810 -nm diode laser for axillary hair removal in Chinese women. J Cosmet Laser Ther. 2016;18:393-6.

25. Chavan D, Chavan D, Nikam B, Kale M, Jamale V, Chavan S. Efficacy of $800 \mathrm{~nm}$ diode laser to treat trichostasis spinulosa in Asian patients. Int J Trichology. 2018;10:21-3.

26. Tulpule MS, Bhide DS, Bharatia P, Rathod NU. $810 \mathrm{~nm}$ diode laser for hair reduction with Chill-tip technology: Prospective observational analysis of 55 patients of Fitzpatrick skin types III, IV,V. J Cosmet Laser Ther. 2020;22:65-9.

Copyright by Sara Gonçalves. This is an open access article distributed under the terms of the Creative Commons Attribution License, which permits unrestricted use, distribution, and reproduction in any medium, provided the original author and source are credited.

Source of Support: Nil, Conflict of Interest: None declared. 